NASAL AND NASO-PHARYNGEAL CONDITIONS AS CAUSATIVE FACTORS IN MIDDLE EAR DISEASES.

BY G. A. LELAND, M.D., BOSTON, MAss.

That the nose is the source of all our woes was once stated as the caption of an article in a New York daily paper. That the nose and nasopharnyx are the source of a great majority of aural woes can hardly be gainsaid. For when we come to inquire into those conditions of the ear which conduce to discomfort, to abolition of the sense of hearing, even to danger to life itself, it will be found that almost all have their morbid origin in this region. For with the exception of certain changes in the secretion of cerumen, which are said to be influenced by naso-pharyngeal circulation, whether exuberant or diminished; also of some markedly virulent cases of external otitis which may possibly pass through a thinned drumhead; and also of trauma, we can exclude largely external conditions as causative factors of middle ear disease.

So also as to invasions from the cerebral side. Trauma, as in fractures of the base, may cause middle ear complications; and, aside from this, may be mentioned that still rarer factor, cerebrospinal meningitis, which may invade the tympanum through the labyrinth. Thus we are confined in our consideration to the protean forms of middle ear diseases which have their origin in extension of inflammation and of infections from the naso-pharynx. And the reason for this extension must be found in loss of the protection normally provided for the safety and the integrity of the conducting apparatus of the organ of audition.

Now the ear is both the most delicate organ of the body, and the best protected. From without, it is fended from damage by a movable cushion, the auricle, set upon the hardest bone in the body, the membrana tympani, its outer limit an inch and a quarter from the surface, approachable in the white races by a narrow curved canal, the inner two fifths of which is of flinty hardness. From within, it is protected by being at the outer end of a tube about one and a half inches in length, whose inner mouth is about three inches from the outside. Thus, by its position, is it guarded from changes of temperature as well as from other sources of injury. Now as most diseases come from the inside, a brief consideration of this protective mechanism may be opportune; and this may be arranged under two heads.

1. Structure of the Eustachian tube, its muscles and surroundings; and their physiological movements.

2. The influence of normal respiration (respiratory movements of membrana tympani).

1. The Eustachian tube is a vertical slit in the side of the naso-pharynx, about one and a quarter inches long, and about one-fourth inch high, whose sides, except at the outer end as it emerges from the tympanum, and at the inner opening in the pharynx, are, when at rest, in high contact. It is lined with columnar ciliated epithelia whose motion is downward towards the pharynx. The posterior wall of the tube consists of a cartilage which curves over at the top so as to form a hooklike projection forward, below the anterior edge of which the anterior wall consists of a fibrous membrane or fascia which is attached to the anterior tip of the Eustachian cartilage continuous with its perichondrium, and, extending downwards, becomes part of the tendon of the origin of the tensor palati muscle. This muscle is the dilator of the tube, and would have been called so if it had not been named before Rüdinger described it and its action. (Bezold.) It has its origin from the anterior tip of the Eustachian cartilage and from the fascia of the anterior wall and then narrows to pass around the hamular process.

Another muscle is the levator palati, which has its origin from the petrous portion of the temporal bone and "its cylindrical belly runs along the floor of the cartilaginous tube (Bezold), to be inserted into the soft palate."

Hovell describes a third muscle of the tube, the salpingo-pharyngeus or retractor of the tube, which, however, is not always present. It arises from the pharyngeal end of the cartilage and passes backward and downward into the muscles of the pharynx.

The action of these muscles is obvious. The tensor palati pulls forward the anterior movable fibrous wall of the tube; the levator palati by its contraction swells its rounded belly upward and pushes upward the floor of the tube, and pushes backward the inner tip of the posterior cartilaginous wall. This action widens the pharyngeal mouth, assisted by the action of the tensor, which immediately follows. This pushing backwards of the tip of the cartilage is assisted by the third muscle, though a weak one.

In normal conditions, therefore, every act of swallowing, most acts of speaking, and even those of respiration, as they cause movements of the very sensitive and flexible soft palate, also cause this continual opening of the inner mouth of the Eustachian tube.

We can conceive, also, that when active contraction ceases and the sides of the tube fall together again to a state of rest, the letting downwards of the floor, as the belly of the levator relaxes, acts as pumping or sucking, to draw air and moisture out of the tube, assisted by the elasticity of the drumhead itself.

Now, the inner opening of the Eustachian tube is situated in the midst of the lateral extension of the adenoidal ring of His or Heryng, and in the anterior wall of the tube between the fascia and the mucous lining is a mass of lymph glands. It will be readily seen that when the naso-pharyngeal glands are hyperplastic and hypertrophied, and the mucous membranes are swolled because of their presence, the normal movements of the Eustachian tube will be curtailed and the circulation increased to even a pathological degree.

Hence the protection of the middle ear afforded by normal physiological ventilation and drainage will be lost. Hence will result rarefaction of the 
air in the middle ear spaces; depression of its only external movable part, the membrana tympani; the possible consequent effusions, serous or mucous, or both, which may cause maceration of the inner walls; and so adhesions, which may take place without inflammation of a sthenic type, without pain or invasion of germs, but producing the insidious variety of adhesive catarrh which leads to loss of hearing sooner or later, becomes the O.M. C. C. of middle and later life, but which is amenable to treatment at any time, but curable only in the earliest stages.

Moreover, if the tube is covered in and pressed upon by adenoidal masses or swollen mucous membranes, so that the action of the cilia of the interior is in abeyance and the pumping motion lost, bacterial invasion may pass up the tube, with so-called extension of inflammatory conditions (O.M.A.; O.M.P.; O.M.P.C.) with all the complications with which we are only too well acquainted.

Both the structure and physiology of these parts point out the rational method of treatment.

2 . One of the greatest factors in the protection which should hedge about the organs of hearing may be found in proper normal respiration, and that means nasal respiration, first, last and all the time. An English writer said long ago that the Eustachian tube begins at the tip of the nose; that meant that the air passages in front of the Eustachian orifices should be free. Mr. Mayo Collier, of London, has shown in an interesting brochure, published some ten years ago, the great influence of nasal respiration upon the integrity of the upper air passages. Others have written on this subject, but as Collier's arguments are especially lucid, they may be mentioned here. There has been much said as to how the accessory cavities of the nose are kept clean, since at least two of these have their only exits near the top of thin, bony cavities (antrums of Highmore and sphenoid), and all of them have only very small exits. Collier shows that this is accomplished by alternate condensation and rarefaction of air, because the nasal orifices are very much smaller than the cavities to which they lead. The air passages, including the nose, pharynx, trachea and lungs, contain a very large quantity of air, and this must be constantly renewed by alternate movements to and fro through the small nasal orifices. In normal quiet respiration, at rest, 20 cubic inches of air must pass through these orifices 20 times a minute. By exercise or exertion this may be increased to 30 cubic inches of air 30 times or more a minute. It is obvious that on inspiration there must occur a partial vacuum behind the introitus nasi, and that on expiration the air pressure must be increased. And it is this alternate variation of pressure which causes an interchange of air through the foramina leading into the accessory nasal sinuses and keeps the orifices clean. In this category may also be considered the middle ear cavity, for its ventilating tube is only somewhat longer than that of the frontal sinus.

That these respiratory movements actually take place was shown by Politzer, in 1860, by the use of his manometer, and has been shown by another European observer who was able to see these movements by covering the membrana tympani with an iridescent paint and using a magnifying glass for accurate observation.

The writer also had an experience which amply proved to his mind that these respiratory movements take place. Some years ago, a laborer was admitted to the City Hospital with large exostoses of the external auditory canal. On account of pain and deafness, caused by accumulation of epithelial débris which could not be removed through the occluded canal, the exostoses were chiseled away after the removal of the cartilaginous and cutaneous canal. There was a perforation through the membrana tympani, and the wound became infected. One morning, on removal of the packing, a bubble was formed over the canal orifice, which swelled out with expiration and was retracted with inspiration, which movements were constant till the bubble burst. His Eustachian tubes were apparently not abnormally open; but this observation and the experiments of others clearly show that there is a movement of air through the Eustachian tube, even though its walls are in apparent contact, and even though the force of the respiratory currents may not be very great.

From the foregoing may be deduced the rationale of treatment. Continuous nasal respiration is essential to the preservation of proper passages through the nose; that is, by the proper circulation of air through this region, proper circulation of the blood and lymph fluid is maintained. Upon this also depends the normal condition of the naso-pharyngeal surfaces, and it is undoubted that the increase in the size of the lymphatic chain which surrounds the upper entrance to the body is largely caused by the lack of the proper play of air through the nose. Slight colds and carelessness of the medical attendants, as well as of the parents, allow the mouth to be used instead of the nose for respiration. Hence the space, not being needed, is filled up by hyperplastic lymph masses called adenoids. The proper movements of the Eustachian tubes are thus curtailed, and thus in earliest life is the foundation laid for deafness after the soft stage of life has passed, that is, after middle life. The time to cure, therefore, O.M.C.C. is before the initial earache has taken place or exudate has formed which causes depression and probably subsequent adhesions in the middle ear, and though in a very limited field there is no part of preventive medicine that can have more far-reaching individual importance than the prevention of these initial earaches in the very young.

To this end, almost universal education must be promulgated to teach people that their children must not be allowed to have earaches, and the general indifference of the community to even a slight earache must be removed, for the middle ear never forgets it. Too many of us, and the writer speaks from personal experience, are suffering from this very neglect which took place in our earliest life; and it is a very serious question 
for the individual when he gets along in life to a position of some importance, at least to himself, if he finds that he is to be shut off from the activities and pleasures of life, more or less, by the inevitable encroachment of almost irremediable deafness which could have been prevented had it been taken in time.

Hence the earliest symptoms of the presence of these growths must be a warning to us for their removal. Slight colds, which are frequent and last a considerable time, should call attention to the ears, and if the drumheads are found to be dull and lusterless, the air in the middle ear having been diminished or lost, the naso-pharynx, and especially the fossa of Rosenmüller, should be immediately cleaned out and nasal respiration insisted upon. But supposing the earaches have already taken place, delays are dangerous at this stage, not only for the present, but more so for the future; and from what has been said, it seems deducible that the rational method of treatment even then is to clear out the naso-pharynx and fossa; that is, to hasten the mechanism of opening and shutting and pumping the Eustachian tube. To this end the sides of the naso-pharynx should be relieved of the growth which is inevitably present, for I am sure there is no child to be seen with earaches not caused by an injury where adenoids are not present, with the possible exception of the infections of the exanthemata and of influenza, and even here it is doubtful.

To possibly throw some light on this point, my assistant, Dr. Henry Tolman, Jr., examined last week 127 cases in the scarlet-fever wards at the Contagious Department of the Boston City Hospital. It is well known that this disease often, if not always, causes an increase in size of these nasopharyngeal lymph tissues. I was surprised to find that he found 43 cases without adenoids. There were 84 cases with adenoids, and of these it is again surprising that there were 72 without aural complications, either past or present. Of the 12 cases with aural complications, acute or chronic, all had adenoids, and of these only 5 had them generally distributed, and the other 7 had them only in the fossæ of Rosenmüller. This bears out my reiterated statement that children with earaches always have adenoid growths, and further emphasizes the importance of clear fossæ of Rosenmüller.

It has been the writer's privilege in numerous cases on the oncoming of earaches to scrape out the fossæ of Rosenmüller and the sides of the nasopharynx at the mouth of the Eustachian tube, and to have others do it under his direction in the hospital. In almost every instance (I recall no exceptions), drainage has been established, inflammation of the ear has gone down, and perforation, pathological or artificial, was not necessary. Of course this procedure, which looks barbarous, cannot be done in every instance. Unfortunately, we have to defer to the opinions, whims and prejudices of guardians and parents, but in those instances where I have been given permission to do it, or where the more formidable ether operation has been allowed, the immediate removal of these growths from the naso-pharynx has been followed by an immediate subsidence of inflammation in the middle ear, both in children and adults.

Hence, with this etiology in view, the rhinologist evidently is the most natural aurist for the beginning of these cases. Now if these cases have been allowed to run on, and the insidious variety of adhesive catarrh has commenced to take place, with or without adhesions, the most rational treatment is that aimed to establish the proper physiological movements of the pharyngeal mouth of the Eustachian tube. From what has been said, it will be recalled that the mouth of this tube is never at rest; that its continuous movements tend to renew the air in the middle ear; but that if the side of the pharynx is swollen, and especially if there is present a mass of adenoidal tissue, the older the tougher, or of adhesive bands or cicatricial tissue in the fossce of Rosenmüller, the pushing back of the tip of the tube by the levator palati and the pulling back by the retractor of the tube cannot take place. Hence the proper movements are curtailed. Formerly, the customary treatment in these cases was by inflation of the middle ear by the Politzer method, or by the use of the catheter; also, by means of tragus massage after Hommel's method, aiming thus to keep up the mobility of the drumhead. In most of these cases lasting improvement did not take place, and could hardly be expected when these grosser methods of inflation were applied only once or twice a week; and it is a question whether the use of Politzer's bag is not injurious because it is usually used with so much force and because these forceful methods produce an overstretching of the membrana tympani and middle ear structures.

The proper method, therefore, in the writer's opinion, is to establish the physiological movements of the air, and to this end must be restored the integrity of the region of the mouth of the Eustachian tube. The fossæ of Rosenmüller must be kept clean and wide, so as to permit the proper excursions of the posterior lip of the tube. Therefore, it may be said that the operation for the removal of adenoidal vegetations, in whatever time of life it is performed, is far from complete and, in fact, utterly useless where adenoids are removed only for the restoration of the respiratory space, and not for the restoration of proper movements at the sides of the naso-pharynx, i. e., utterly useless as far as the ear is concerned.

Moreover, one result of the operation in this fossa, which is a sulcus narrowing at depth outwards, and which by the rhinoscope may appear but one-fourth inch deep, but which, by the use of the finger, may be found to be one-half inch or more in depth, is to wound its surfaces. Hence, as these surfaces are movable, there is a tendency for the production of adhesions, so that sooner or later after the operation this fossa will be found to be occluded by fibrous tissue or by a reproduction of adenoids which it is a fact are absolutely impossible of complete removal, especially in this locality. Hence, in order to keep these fossæ clean so to insure their proper movements, 
it is occasionally necessary to scrape them after the major operation and to make application of astringents in them soon and perhaps frequently afterwards to keep the adhesions from forming.

Now it is evident that such subsequent treatment is difficult of accomplishment; but it is also evident that in many cases it will be necessary, if the proper movements of this tube are to be established and hearing preserved; that it is without doubt much better to suffer a few weeks of uncomfortable treatment than that the rest of life after middle age or earlier should be compromised by increasing deafness.

But the obliteration of this fossa, more or less complete, is not confined to early life. It frequently obtains also in adults with increasing deafness, and compromises the proper movements that establish ventilation of the middle ear. It seems to the writer much more rational to reestablish proper movements of these parts whereby the patient treats himself by the motions of swallowing, and by movements of respiration twenty to thirty times a minute, than it is to rely wholly on the use of the catheter or Politzer's bag. I beg to submit that this auto-treatment is much more effective where the middle ear has not been too firmly bound down by adhesions than any other treatment which can be artificially applied. To emphasize this position might be cited numerous cases which have been formerly under treatment for many years, where the reestablishment of these palatal and respiratory movements has brought the ear back to a satisfactory degree of hearing and given the patient much greater comfort and a brighter outlook on life.

In one case, a man sixty years of age, where a diagnosis had been made of anchylosis of the stapes and the patient, after twenty-five years of treatment, had been given over to that hopeless state where further treatment was considered useless, the establishing of these movements brought back hearing so that it was considered by his family that he had been restored to his normal condition.

Another case of fifty-eight years of age, after the same length of former treatment, had been given over to occasional observation without treatment, which was considered hopeless interference.

The hearing has been restored from 1 inch right ear and $\frac{1}{2}$ inch left to between 1 and 3 feet right ear and $\frac{1}{2}$ to 1 foot left, for Politzer's acoumeter. Here, general conversation at the table had been wholly lost, but now there is comfort at the table and in the office, which had been unknown for years.

Another one, a man of sixty-four years of age, after forty years of ordinary methods of treatment, finally consented to have his nose restored, the swellings of his naso-pharynx reduced, and so was brought into a condition where intercourse with his fellow men has now become a comfort when formerly it was a trial.

The reason which may be given for this difference in these cases is deduced from what has been said above, and here reiterated, that the proper respiratory and naso-pharyngeal movements are operative twenty-four hours in each day, twenty or more times a minute, and that these very gentle movements of the drumheads tend to stretch, or make flexible, the stiffened membrane and even the adhesions, just as continual dropping of water may wear away the rock. In the writer's opinion, this rational method of treatment has given better results in cases of encroaching deafness than are produced in any other way; and that in many cases this treatment must be insisted on at the very beginning of life.

There is one form of disease in which as yet very little progress has been made, viz., oto-sclerosis. May it not be true that even this disease started by inflammatory attacks in early life, influenced by rheumatism and other dyscrasiæ, may be prevented with all the rest by rational treatment of the naso-pharynx before these inflammatory attacks are begun?

Dependent on the nose and throat may be mentioned several isolated symptoms. And the first is pain. Many patients come to us complaining of earaches, where, after careful examination, there is no evidence of inflammatory mischief in the middle ear or its annexes. This pain is, therefore, reflex and may come from various locations, even from the aryepiglottic folds or from the sides of the larynx, as was shown many years ago by our colleague, Dr. J. Orne Green. Most frequently it comes from the tonsils, radiating upward. It occasionally comes from swelling of the lateral pharyngeal lymphatic bands behind the posterior pillars of the fauces, which pass up around the region of the Eustachian tubes. It may come from a tooth, painful because of decay or improper striking, for any part of the trigeminal in its myriad branches may have the cause of pain which may be referred to any other part. Also, we may have pain in the ear from intranasal pressure, from suppuration of the sphenoid, and probably also the ethmoid; but this only shows that in some cases the whole periphery, or whole nervous supply, must be overhauled to find the cause.

The second symptom of this nature is a feeling of fullness in the ear, as if there were a plug of cotton, the removal of which would clear up this condition of discomfort. I have found this pressure feeling to be only a lesser degree of that which means pain, and to be caused in a large number of cases by pressure of the corresponding second turbinal upon the top of the third, or on the outer wall. The removal of this pressure clears up the feeling of plugging, and it is utterly irrational to inflate the ear in these cases, for in most of them hearing tests will prove that, the ear being all right, there can be no cause for the sensation there.

The third symptom to which I would like to call attention is tinnitus, and especially the low-toned roar, often with pulsations, or, as the patient describes it, a hammering. In these cases there is frequently an effusion in the middle ear, but as often the tympanum is clear. That this variety is often due to hyperemia of the lymphatic structures in the sides of the naso-pharynx, and 
especially in the fossæ of Rosenmüller, has been abundantly proved by its almost immediate cessation after they have been cleared out and kept clean by astringent applications, often necessarily made with considerable force. So, also, the hissing variety of tinnitus, especially in late or even middle life, is often thought less surely diminished or abolished by reduction of the circulation around and inside of the mouth of the Eustachian tube. And to this end, for a result more or less permanent, let it again be reiterated that normal unobstructed nasal respiration must be established, and further that that most common of all nasal obstructions corrected, viz., the vicious habit of mouth breathing.

The fourth and last symptom is vertigo. The occlusion of the nose with its hypercirculation is occasionally responsible for this symptom, even without active aural lesions. An old habitué of the aural service many years ago is well remembered who for several months came to the clinic led by his daughter because of staggering in the street. He was treated aurally by me three to four months with little relief, by my colleague for the next six months, and was still coming in the same way when the service was changed. Then it was discovered that his turbinates, lower and middle, were much engorged. On reduction of these bodies, the vertigo ceased and hearing improved. This case has served good stead as a pointer to occasional cases of nasal vertigo.

In speaking of these functional movements, those produced by blowing the nose have not been mentioned because it is considered, together with the Valsalvan experiment, a pernicious practice, resulting too often in over-stretching of the membrana tympani and of the middle ear tendons and ligaments, and because proper treatment and proper use of the nose should make the use of the handkerchief unnecessary, for there is no doubt that this act increases congestion and intensifies most morbid conditions.

In closing, it must be conceded that the whole subject has by no means been touched upon in this paper - it is too large for the time allotted; that many of the diseases of the ear, of inflammatory and infectious extensions, have been only hinted at, but it has been attempted to call attention to certain principles which have been of value as stimuli in practice, and to certain methods which have been followed by satisfactory results. Nor is it thought for a moment that anything new has been brought forward, anything outside of the practice or experience of most, if not all, of this audience of specialists; but only that this paper is a small part of this symposium which, with its discussion, is to go out to do missionary work among the family physicians, and especially the pediatrists, and even the obstetricians, who have to do with the youngest victims of these troubles, when treatment is most effective for the future as well as for the present; and also to make clear that treatment of the naso-pharynx, especially operative treatment, is in the province of the aural specialist, since its effect on the function of hearing is of the utmost importance.

\section{THE DIAGNOSIS OF PHTHISIS, AND ITS ASSOCIATION WITH CERTAIN SIMULATING DISEASES.*}

\author{
By Cleaveland Floyd, M.D., \\ WILLSTON W. BARKER, M.D., BOSTON, M.ISS.
}

CERTAIN FREQUENTLY NEGLECTED POINTS IN THE DIAGNOSIS OF PHTHISIS.

The diagnosis of phthisis in its incipiency presents to the great body of medical men one of the most difficult problems. No disease is so multiform in its manifestations or so often obscured or simulated by other diseases. Aside from these facts, the location and the depth of beginning pulmonary involvement are of great importance in determining the ease with which a diagnosis can be made. The small lobule of tuberculous tissue is almost impossible of detection on account of its minute size, and even where several have coalesced, compensatory emphysema precludes detection. Again, where the primary area of consolidation occurs in those parts of the lung away from the surface, normal lung tissue coming between the diseased area and the ear will exclude all transmission of abnormal adventitious sounds. In such cases, phthisis may not be suspected until routine sputum examination shows tubercle bacilli to be present. Furthermore, in those cases whose pulmonary expansion is limited and areas of atelectasis are constantly present at the margins of the lung, this condition may obscure a small area of caseous bronchopneumonia in this location. The phthisical patient, by the very reason of his general physical condition, is more prone to develop complicating diseases, and asthma, chronic bronchitis or nephritis add one more element of difficulty in the way of the early detection of phthisis.

For this and many other reasons, an early diagnosis of pulmonary tuberculosis consists of a summary of all factors obtainable as indicative of its presence. Far too many physicians neglect to treat a patient for phthisis until they have proved its presence in the individual. Treatment may, with advantage, be instituted while the suspicion of phthisis is being proved a certainty.

The onset of phthisis is varied and often ushered in by some complicating disease whose presence apparently accounts for all symptoms. Bronchitis, laryngitis or marked debility are often the beginnings of phthisis and their signs or symptoms may be all that are obtainable for weeks or months. Nevertheless, their association with pulmonary tuberculosis is generally appreciated and the accompanying lung condition, therefore, closely scrutinized. Hemoptysis is of immense diagnostic significance, even in the absence of pulmonary signs, and while it may not necessarily arise from tuberculosis, its occurrence is always suggestive of tubercle formations in the lungs. Not infrequently phthisis first produces marked constitutional symptoms by gastric upset. Stomach cough is a well-known expression. The Society, June 15, 1909. 